\title{
Shroud like coloration of linen, conservation measures and perception of patterns onto the Shroud of Turin
}

\author{
Paolo Di Lazzaro a and Daniele Murra \\ ENEA, Dept. Applications of Radiation, Via E. Fermi 45, 00044 Frascati, Italy
}

\begin{abstract}
We present new experimental results on ultraviolet laser processing of linen fabrics that complete and update the previous studies at the ENEA Research Centre of Frascati seeking a coloration mechanism able to reproduce the microscopic complexity of the body images embedded onto the Shroud of Turin. The achievement of a Shroud-like coloration of linens by using vacuum ultraviolet light pulses allowed to recognize the main photochemical reactions possibly involved in the coloration process. The identification of these reactions offered hints for long-term conservation measures of the body images on the Shroud of Turin. Finally, we discuss how image processing of photographs of the Shroud may cause misleading effects, which led some researchers to perceive, e.g., inscriptions and a second face on the backside of the cloth, which are unlikely to be on the Shroud, confirming that there is a narrow boundary between image enhancement and manipulation.
\end{abstract}

\section{Introduction}

The odd chemical and physical characteristics of the image of a scourged man on the linen cloth of the Shroud of Turin (TS) make difficult creating an image that matches (or even approaches) its peculiar superficiality and chemistry at the microscopic level. After the most recent in-depth experimental analysis of the images on the TS in 1978, the team of the Shroud of Turin Research Project (STuRP) wrote in the official summary of their final report: "We can conclude for now that the Shroud image is that of a real human form of a scourged, crucified man. It is not the product of an artist. The blood stains are composed of haemoglobin and also give a positive test for serum albumin. The image is an ongoing mystery and until further chemical studies are made, perhaps by this group of scientists, or perhaps by some scientists in the future, the problem remains unsolved." [1].

The above conclusion was a consequence of the on-site analyses made by microscopy, visible ultraviolet and infrared spectrometry, X-ray fluorescence spectrometry, and thermography. Later observations on linen fibres and threads sampled from the TS were made by microchemistry, petrographic microscopy, scanning-electron microscopy, energy dispersive X-ray analysis, pyrolysis-mass-spectrometry, and laser-microprobe Raman analyses [2, 3]. In particular,

- reflectance spectra, chemical tests, laser-microprobe Raman spectra, pyrolysis mass spectrometry, and X-ray fluorescence all show that the image is not painted with any of the expected, historically-documented pigments and media.

\footnotetext{
${ }^{a}$ Corresponding author: paolo.dilazzaro@enea.it
}

This is an Open Access article distributed under the terms of the Creative Commons Attribution License 4.0, which permits unrestricted use, distribution, and reproduction in any medium, provided the original work is properly cited. 
- both kinetics studies and fluorescence measurements support the image was formed by a lowtemperature process. As a consequence, it is unlikely a Shroud-like image can be produced by contact with a heated statue, as discussed in [4];

- the coloration depth is extremely shallow, in the range $200-600 \mathrm{~nm}$. Recent measurements on image-fibres of the TS suggest that the coloration depth is $200 \mathrm{~nm}$, which corresponds to the thickness of the primary cell wall of each linen fibre [5];

- the coloration process of the TS image involves oxidation, dehydration and conjugation of polysaccharide structure of fibres, to produce a conjugated carbonyl group as the chromophore;

- the microscopy images show the so called "half tone effect", that is, shading of the body images is not accomplished by varying the colour, but by varying the number of colour fibres per unit area at the microscopic level. In other words, the image seen at the macroscopic level is an areal density image. As the diameter of each fibre in the Shroud's yarns ranges between 15 and 30 micrometers, the half tone effect suggests that the image cannot have been hand-coloured, unless the artist had a micrometer-size brush and a microscope;

- blood tests positive for haemoglobin, serum albumin and immunoglobulin. The anatomical consistency of blood and serum versus wounds, including typical halo fluorescence around the main bloodstains, would be consistent with a haemolytic process caused by torture.

All the countless attempts to create a Shroud-like image since the Pia's photographs in 1898 have failed to adequately reproduce the above characteristics all together. Some researchers have obtained coloration and/or images that look similar [6] but no one has created images that match both microscopic and macroscopic features of the TS image. The answer to the question of how the image was produced or what produced the image is still unknown. After, the inability to replicate the microscopic complexity of the image on the TS prevents formulating a reliable hypothesis on the process of the image formation.

In this paper we present the survey of the overall research work on Shroud-related issues done during the years 2005-2013 by the Excimer Laser Laboratory at the ENEA Research Centre of Frascati [7]. Section 2 summarizes our attempts to find a photochemical coloration mechanism able to reproduce the microscopic complexity of the body images on the TS. Our identification of the specific groups and reactions involved in the laser coloration process offered hints for long-term conservation measures of the TS, as discussed in Sect. 3. Finally, we made a study of the misleading effect of deep image processing of photographs of the TS, as detailed in Sect. 4.

\section{Laser irradiations of linen fabrics at ENEA}

It is well known that the energy carried by short-wavelength radiation breaks the chemical bonds of the irradiated material without inducing a significant heating: we say short-wavelength radiation generates a "photochemical reaction". Moreover, linen has a molar absorptivity which increases when decreasing the radiation wavelength: the smaller the wavelength, the thinner the material necessary to absorb all the radiation. Then, we chosen the ultraviolet (UV) radiation to obtain at least two of the main characteristics of the TS image: a thin coloration depth and a low-temperature image-formation.

We first measured the absolute reflectance spectra of modern linens we have used in our experiments. These linen fabrics were hand-made in the period 1936-1950 and never used before, The results showed a lower reflectance of our linens vs. the TS in the spectral region $520 \mathrm{~nm}-600 \mathrm{~nm}$, confirming our linens are less yellowish than the TS, as expected because of the different age and different aging-related oxidation and dehydration. However, we measured a perfect overlap of our linens with the absolute reflectance of the TS in the UV and vacuum UV (VUV) spectral regions, [8]. Thus, when irradiated in the UV and VUV, our linen fabrics behave like the linen of the TS. 

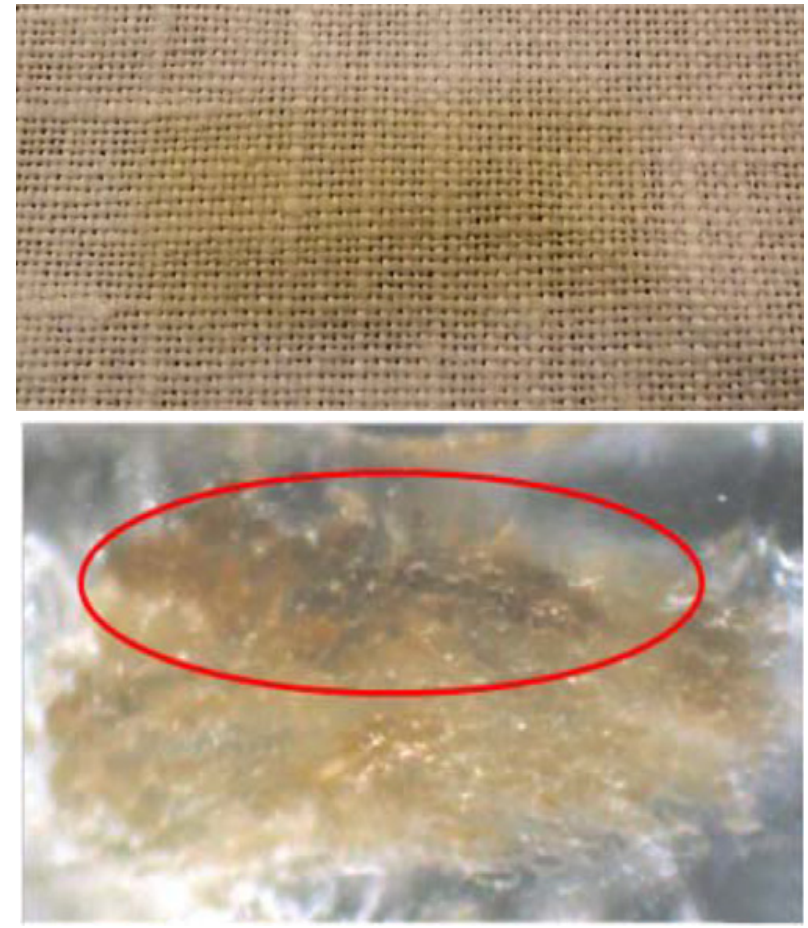

Figure 1. Above: faint linen coloration after 10 laser shots at $308-\mathrm{nm}$. Fluence/shot $0.15 \mathrm{~J} / \mathrm{cm}^{2}$. Intensity/shot $4.5 \mathrm{MW} / \mathrm{cm}^{2}$. $1 \mathrm{~Hz}$ repetition rate. Source: ENEA. Below: cross section of a linen yarn irradiated at $308 \mathrm{~nm}$, contrast enhanced. The red ellipse shows the depth of the coloured region. From [10].

\section{1 $\mathrm{XeCl}$ excimer lasers irradiations}

At the beginning we irradiated linens by an $\mathrm{XeCl}$ excimer laser facility named Hercules, which emits UV 308-nm laser pulses, each one lasting $120 \mathrm{ns,} \mathrm{without} \mathrm{obtaining} \mathrm{any} \mathrm{coloration} \mathrm{results.} \mathrm{Namely,} \mathrm{linens}$ irradiated with high fluence/intensity were carbonized, while at intermediate and low fluence/intensity values we did not observe any change of the linen threads, both at macroscopic and microscopic level. These bad results were in agreement with the analogous failure at Los Alamos National Laboratories (LANL) using 50-ns long excimer laser pulses [9].

Then, we irradiated the linen by using another XeCl laser (LPX 305) emitting pulses 4 times shorter and with an energy per pulse 12 times smaller than the Hercules laser, at the same $308 \mathrm{~nm}$ wavelength. In this configuration we achieved a permanent coloration of linens in a narrow range of pulse duration, intensity, number of laser pulses and time interval between successive pulses [10]. This coloration was superficial at the thread level, see Fig. 1.

In this way, we have shown that the duration of the UV light pulses plays a main role in the photochemical reactions producing linens coloration, and that the experimental failure experienced at LANL using excimer lasers [9] was due to parameters (in particular the laser pulse-width) outside the narrow range of values able to generate the linen coloration.

\subsection{ArF excimer lasers irradiations}

In order to obtain a thinner coloration depth than that obtained by the $\mathrm{XeCl}$ laser, we have used the VUV radiation emitted by an ArF excimer laser (emission wavelength $193 \mathrm{~nm}$, pulse-width $12 \mathrm{~ns}$ ). In this 


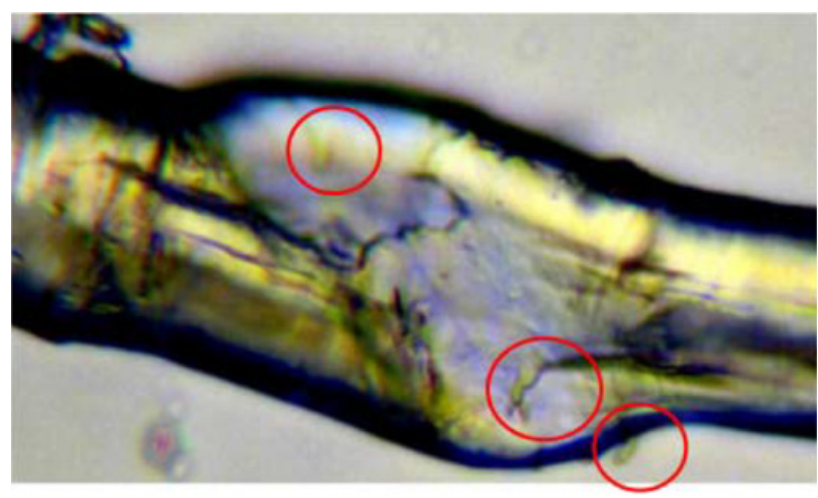

Figure 2. Microscope view of a single linen fibre mechanically damaged after it was coloured by 193-nm laser irradiation. Circles show pieces of the broken 200-nm-thin external layer of the fibril (the so called "primary cell wall"). Note these pieces are yellowish, compared with the uncoloured medulla (secondary cell wall) of the fibre. From [11].

respect, our experiments were successful, as we obtained a permanent coloration of the outside fibres of the linen threads [8], and using a particular set of irradiation parameters we obtained a coloration limited to the primary cell wall of the linen fibres $[8,11]$, that is, a coloration superficial at the fibre level similar to that of the TS [5], see Fig. 2.

In addition, we observed the hue of yellow colour slowly changes with the number of consecutive laser pulses, thus allowing an accurate control of the RGB value within $2 \%$ by selecting the total irradiation fluence/intensity [11], e.g., by varying the number of laser pulses.

\subsection{Excimer laser coloration vs. Turin Shroud coloration}

The linen coloration is a threshold effect for both $308 \mathrm{~nm}$ and $193 \mathrm{~nm}$ irradiations, i.e. the colour is obtained only when the laser fluence/intensity on the linen is above a given level. However, after laser irradiations just below-threshold that at first do not generate a visible coloration of linen, a latent coloration appears either by artificial or natural aging of linen, as detailed in $[8,10,11]$. Latent coloration is interesting for the synergy between radiation and the oxidative-dehydrating effect of aging which trigger the coloration process, and for historians, attracted by the possibility that, whatever has caused the TS image, the coloration may have "developed" over time. Latent coloration can be explained by oxidation and dehydration of the cellulose (caused by heat or by natural aging) amending the new chemical bonds induced by laser irradiation, thus facilitating the formation of conjugated unsaturated structures that are essential part of the photochemical reactions involved, as detailed in [11]. The synergy between heat and UV light on cellulose is discussed in [12], showing how the process initiated by UV radiation is accelerated and reinforced by heat.

The partial inhibition of fluorescence induced by both UV and VUV laser radiation shown in $[8,10,11]$ is an additional feature of our coloration similar to the TS image.

Excimer laser irradiations of linens activate a "cold" photochemical coloration process. In fact, we have experimentally measured a thermal heating of few degrees centigrade after UV and VUV irradiations [11] which is irrelevant compared to colouring linens by scorching. In addition, the spectral energies of single UV and VUV photons (4 eV for $308 \mathrm{~nm}$, and $6.4 \mathrm{eV}$ for $193 \mathrm{~nm}$ ) are higher than the energies of most chemical bonds in cellulose, thus allowing the direct breaking of the same bonds and the creation of new chemical bonds with a negligible secondary heat generation. Photochemical reactions fit the "cold" coloration process of the TS estimated by STuRP [2]. 


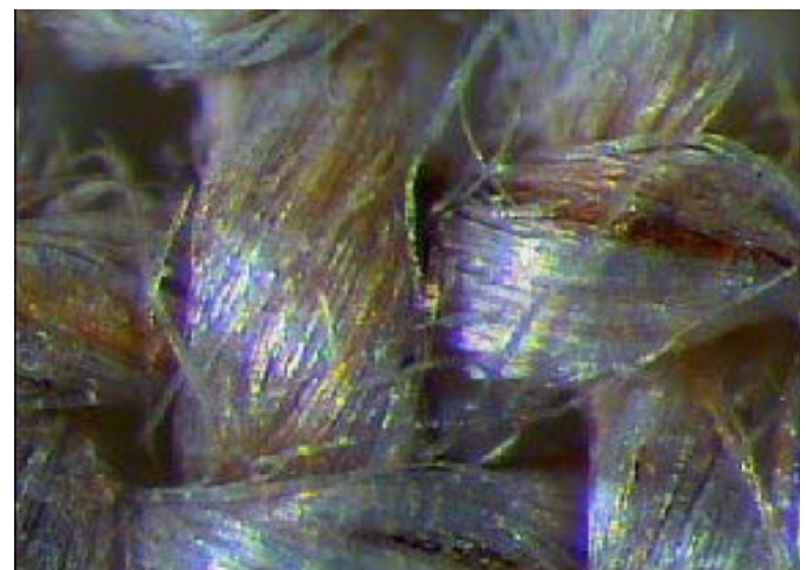

Figure 3. Microscope view of linen threads after VUV laser irradiation. Single colored fibers are visible next to uncoloured fibres, like in the Shroud image. From [11].

The TS image seen at the macroscopic level is an area-density image [2]. In other words, shading is accomplished by varying the number of colour fibres per unit area, and not by varying the colour of the fibres. At the usual observation distance (larger than $2 \mathrm{~m}$ ) our eye-brain system cannot distinguish the single fibre and it makes a spatial average of coloured/uncoloured fibres to perceive the image shading. Occasionally, we found these features in our irradiated linens, see Fig. 3, which shows coloured next to uncoloured fibres in the same thread. However, we did not fully achieve a "half tone effect" comparable to that observed on the TS [11].

One of the most interesting results we achieved is related to the different hue of colour obtained by UV and VUV radiation, namely dark yellow - light brown and yellow, respectively. We remind that light brown has RGB values very close to yellow ones: to some extent, light brown is a sort of low-brightness yellow. As a consequence, the apparently huge difference between yellow and brown is more due to a perception process than to an effective RGB difference.

In any case, the achievement of light brown linen coloration vs. yellow linen coloration is due to different chains of photochemical reactions respectively triggered, and this difference may be used to seek for the specific groups and photochemical reactions involved. In particular, the VUV radiation at $193 \mathrm{~nm}$ is preferentially absorbed by alkenes $(-\mathrm{C}=\mathrm{C}-)$ and ketonic carbonyl $(-\mathrm{C}=\mathrm{O})$ groups, which are typically abundant in cellulose and hemicellulose of the primary cell wall of linen fibres. This absorption may trigger a reaction chain which leads to photo-oxidation (aging) and to new alkenes and carbonyl groups. After a proper irradiation dose, new conjugated $\mathrm{C}=\mathrm{C}$ and $\mathrm{C}=\mathrm{O}$ groups are formed, thus shifting absorption band to longer wavelengths, namely in the blue-green spectral region. The increasing absorption of blue-green finally produces the yellowish Shroud-like coloration. A detailed discussion of this important issue can be found in [11] and references therein.

\subsection{Shroud-like laser drawing}

Once known the narrow-range values of laser fluence (energy/area) and intensity (peak power/area) able to generate a Shroud-like coloration, we can adjust the laser energy to a value suitable to obtain a linen coloration over a focused laser spot having an area of few $\mathrm{mm}^{2}$. By using this small laser spot like a bristle brush, we can draw a figure moving this "laser-brush" with respect to a linen fabric. As an example, we loaded the bitmap image of the logo of the 2010 Shroud exhibition in our PC. We chose the overall size of the logo by adjusting the number of horizontal and vertical pixels. Each pixel 

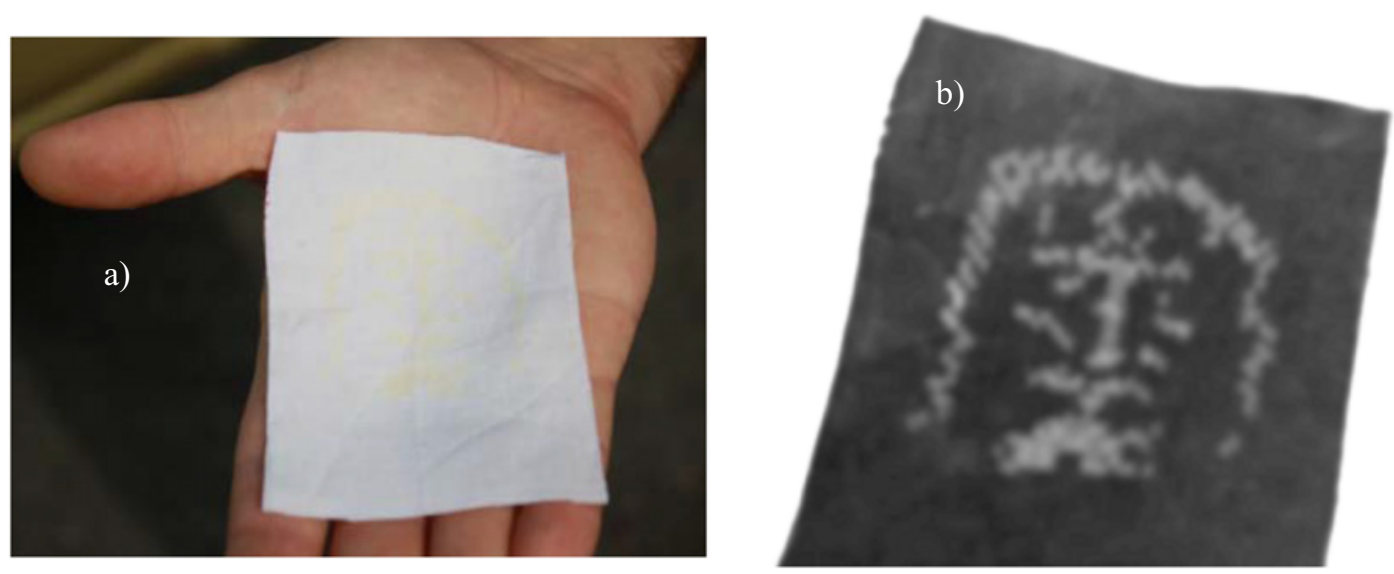

Figure 4. a) Faint yellow image of the face of the logo of the 2010 Shroud exhibition drawn on linen by using the excimer laser beam as a contactless brush. Color is normalized to the reference white. b) Negative of the same image in a). Source: ENEA.

corresponds to the size of our "laser-brush". The linen was put on a frame placed on a XY double translation stage with a maximum stroke of $200 \mathrm{~mm}$ and one micrometer accuracy of repositioning [13]. A dedicated software allowed to move the double stage according to the bitmap of the logo and to trigger the laser shots just when the stages reached a given point corresponding to the colour pixel. In less than 10 minutes we have drawn the $50 \mathrm{~cm}^{2}$ face shown in Fig. 4a. Note the yellowish image is extremely faint and difficult to see, showing a low-contrast similar to that of the TS image. On the contrary, the negative of the same image shows a clearly visible face, and even the single laser-shot-pixel can be identified, see Fig. $4 b$.

In this preliminary attempt, we did not pay attention to the image shading, having reproduced a logo which has a binary coloration. However, a shaded image can be obtained simply reducing the $\mathrm{XY}$ movement between successive laser shots to be less than one pixel, that is, less than the laser spot diameter. Obviously, the laser fluence of each shot must be reduced accordingly.

The linen in Fig. 4 is presently exposed at the Museum of the Shroud in Turin http://www.sindone.it/\#museo_tour\&LL=en.

\section{Conservation measures}

The irradiation results briefly summarized in Sect. 2 show a dramatic capability of UV and VUV radiation to modify and age the cellulose of linens. In particular, by using a petrographic microscope we have observed some defects induced by UV radiation in the structure of irradiated linen fibres, similarly to very old linen fabrics [10]. We can therefore infer that UV pulses change the crystalline structure of cellulose in a similar manner as aging and low-intensity natural radiation accumulated in a long period do. In this respect, the knowledge of the photochemical processes able to generate a Shroud-like coloration, as outlined in Sect. 2.3, is essential to plan the long-term conservation of both the cloth and the image on it.

We have identified Radon ( $\mathrm{Rn})$ as the most dangerous source of natural radiation, potentially affecting the long term conservation of the image contrast and visibility. In fact, at sea level Rn (an invisible, odourless, radioactive gas formed by the disintegration of Thorium and of Radium) is the main source of natural radiation. In fact, $\mathrm{Rn}$ emits alpha particles and produces several solid radioactive products called Rn-daughters, which in turn emit beta-particles and ionizing radiation. 
Table 1. Our suggestions of the optimum physical parameters and measures aimed at a long-term conservation of the Shroud, vs. the available characteristics of the reliquary made by Thales Alenia Space, where the Shroud is presently conserved. From [14].

\begin{tabular}{|l|l|l|l|l|}
\hline & $\begin{array}{l}\text { Gases and } \\
\text { pressure }\end{array}$ & $\begin{array}{l}\text { Relative } \\
\text { humidity, } \\
\text { temperature }\end{array}$ & $\begin{array}{l}\text { Box material / } \\
\text { thickness } \boldsymbol{\text { Radon }}\end{array}$ & issue \\
\hline $\begin{array}{l}\text { Our } \\
\text { suggestions }\end{array}$ & $\begin{array}{l}99.6 \% \mathrm{Ne} \text { or } \mathrm{Ar}, \\
0.4 \% \mathrm{O}_{2}, \\
9 \mathrm{mbar} \text { water } \\
\text { vapour, } \\
\mathrm{P} \geq 1 \mathrm{bar}\end{array}$ & $\begin{array}{l}\mathrm{RH} \approx 40 \% \\
\mathrm{~T}=20^{\circ} \mathrm{C}\end{array}$ & $\begin{array}{l}\mathrm{Al} \text { or Al-based } \\
\text { alloy } \\
\mathrm{t} \geq 5 \mathrm{~mm}\end{array}$ & $\begin{array}{l}\text { Building materials } \\
\text { and objects around } \\
\text { must be Radon-free }\end{array}$ \\
\hline $\begin{array}{l}99.5 \% \mathrm{Ar}, \\
\text { Present } \\
\text { reliquary }\end{array}$ & $\begin{array}{l}0.5 \% \mathrm{O}_{2} \\
\mathrm{P} \text { equalised to the } \\
\text { atmospheric } \\
\text { pressure }\end{array}$ & $\begin{array}{l}\mathrm{RH}=50 \% \\
\mathrm{~T}=19^{\circ} \mathrm{C}\end{array}$ & $\begin{array}{l}\mathrm{Al} \text { alloy, } \\
\mathrm{t} \text { is variable }\end{array}$ & $\begin{array}{l}\text { Information not } \\
\text { available }\end{array}$ \\
\hline
\end{tabular}

In [14] we have suggested simple actions to moderate the Rn-associated risk level, and we evaluated the optimum conditions aimed at a long term conservation of the TS. As summarized in Table 1, the TS should be conserved in a few millimetre-thick Aluminium $(\mathrm{Al})$ or Al-based alloy box, filled with a proper mixture of high-purity $\mathrm{Ne}$ or $\mathrm{Ar}$ gas, $\mathrm{O}_{2}$ and water vapour, in order to set an equilibrium between the dehydration and oxidation of linen fibres to maintain the image contrast and visibility, while avoiding the growth of anaerobic organisms. The total pressure should be just above the atmospheric pressure to prevent air and dust from entering inside the box. A constant temperature of $20^{\circ} \mathrm{C}$ eliminates any risk of autocatalysis-like processes from the acidic structures produced by previous oxidative activity. The Al box containing the TS should be located in a building made of Rn-free materials, and every object, treasure, furnishing, floor, wall made of Rn-reach materials like tuffaceous rocks, porphyry, basalt, gneiss, syenite or granite must be removed from around the reliquary. The amount of $\mathrm{Rn}$ in the room should be routinely monitored.

\section{Misleading image processing}

Another issue studied at the ENEA is the potentially misleading effect of software techniques used to elaborate photographs of low-contrast stains on the TS. Several works have proposed interpretations of patterns on the TS that become visible only after the use of image processing tools, including presumed inscriptions [15], coins [16] and flowers [17], as well as a face-like image perceived on the backside of the TS [18]. Our results suggest that these interpretations cannot be considered self-consistent proofs. In particular, in [19] we have shown that image processing of recent, high-resolution photographs of the TS may lead some to perceive inscriptions that are unlikely to exist. This is because our brain' ability to retrieve incomplete information [20] acts in synergy with the pareidolia effect [21] to connect and interpret false image pixels which are created after contrast enhancement and luminosity adjustment. At the end of this elaboration of our brain, we perceive patterns that "make sense" but do not necessarily exist in the original image. In addition, we have shown for the first time that the alleged hidden image of a face on the backside of the TS reported in [18] has a very poor spatial correlation with the corresponding face image on the frontal side of the TS [19]. More in detail, Figs. 5a and 5b show the negative of the "hidden face" revealed after digital image processing of a screened halftone photographic reproduction of the backside of the TS taken from a book, compared with the negative of the face of the TS.

We checked both Figs. 5a and 5b have almost the same histogram of "number of pixels" vs. "gray levels", see Fig. 6. 

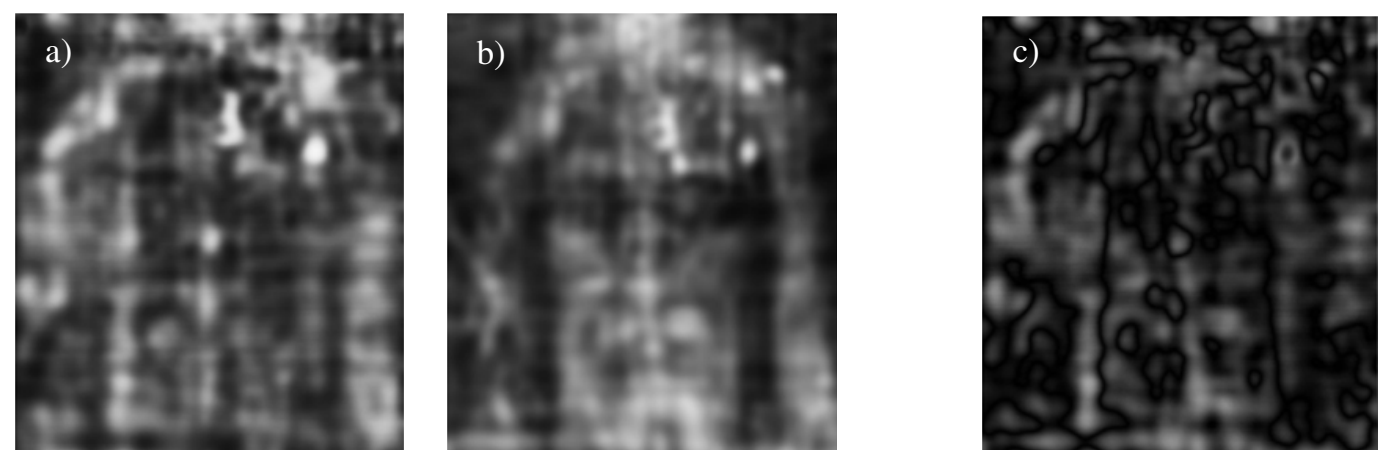

Figure 5. a) Negative of the alleged hidden face on the underside of the Shroud made visible by deep image processing of a photograph printed in a book. The image is inverted right-left. b) Negative image of the face visible on the frontal side of the Shroud, elaborated as well as a). From [18]. c) Image resulting from digital subtraction of the values of the gray levels of a) and b) after a careful overlap of images a) and b) using blood marks as a reference. From [19].

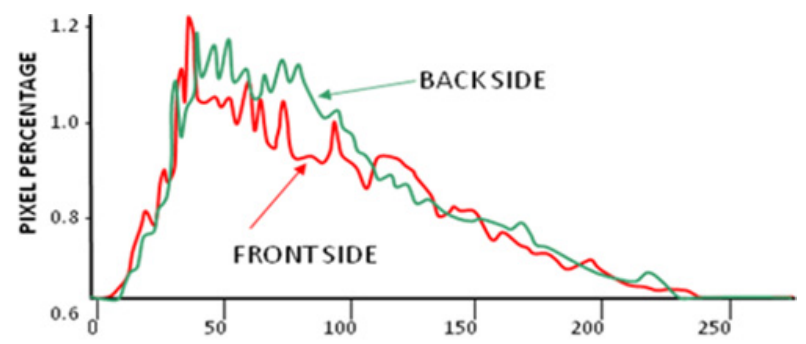

Figure 6. Pixels distribution vs. gray level of the face on the front (Fig. 5b) and on the back (Fig. 5a) side of the Shroud. The number of pixels for a given gray value is almost the same in the two cases. Elaboration: ENEA.

As a consequence, after the optimum spatial overlap of Figs. 5a and 5b (using bloodstains as a reference), the absolute value of the difference of gray levels between Figs. 5a and 5b should give a black figure when the two figures are perfectly correlated, or a noisy figure when they have a partial degree of correlation, or a recognizable face when the correlation is poor. The result of this pixel-bypixel calculation is shown in Fig. 5c, where a Shroud-like face can be still recognized, thus revealing the lack of spatial correlation of Figs. 5a and $5 \mathrm{~b}$ as a whole.

The lack of spatial correlation between the "hidden" face underside and the face on the TS is confirmed by a template matching based on a cross-correlation algorithm that calculates the Pearson product-moment correlation coefficient $S$ according to the following equation:

$$
S=\frac{\sum_{i j}\left[\left(A_{i j}-<A>\right) \cdot\left(B_{i j}-<B>\right)\right]}{\sqrt{\sum_{i j}\left(A_{i j}-<A>\right)^{2} \sum_{i j}\left(B_{i j}-<B>\right)^{2}}}
$$

where Aij (Bij) is the gray level of the single pixel (i,j) of the image $\mathrm{A}(\mathrm{B})$, and $\langle A\rangle(<B>)$ is the mean value of the gray level of the image A (B).

When applying Eq. (1) on the whole images in Figs. 5a and 5b, we obtain $S=0.4$, which is a very poor spatial correlation value, as detailed in [19].

Note that the Pearson correlation coefficient $S$ in Eq. (1) is the same that was used in [18] to show a good correlation between the face on the TS and the alleged hidden face on the back of the TS. 


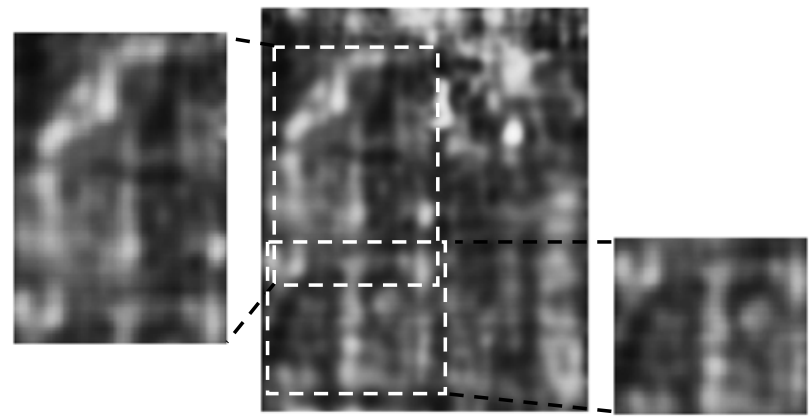

Figure 7. Middle: the presumed hidden face as in Fig. 5a. Left: a Shroud-like face we perceive in the top-left part of Fig. 5a (corresponding to the rectangular inset). Right: another face we can perceive is in the bottom left part of Fig. 5a (rectangular inset). Looking carefully, we may recognize a bear-like muzzle in the up-right corner of Fig. 5a. These examples show how it is easy to deceive our perception, due to our brain' ability to interpret false pixels, that allow us to see faces and other figures in Fig. 5a that do not exist. Elaboration: ENEA.

The reason why the very same Eq. (1) gave a correlation $S>0.7$ in [18] and $S=0.4$ in [19] is that in [18] $S$ was calculated across small areas of the face (e.g., tip of the nose, part of the moustache, etc.) where it is easier to find random correlations between the Fig. 5a and Fig. 5b. In fact, it is well known that when comparing two different figures, the smaller the areas you analyze and compare, the higher the probability to find some degree of spatial correlation. However, the two figures remain different, independent of random correlation over small regions.

We recognize that our results showing the lack of spatial correlation between Figs. 5a and 5b, are just confirmations of the results of Prof. Balossino [22], showing that the Fourier transform of a highresolution image directly obtained by in-depth scanning of the backside of the TS, did not show any face or any other image. Clearly, the direct analysis on the underside of the TS in [22] has a greater reliability with respect to the digital processing of a photograph printed in a book in [18].

It remains the problem to understand why many of us perceive a Shroud-like face in Fig. 5a, if it does not exist. In [19] we have shown that the face-like pattern we perceive may be due to a joint effect of the brain's ability to supply the missing contours in order to make sense out of any pattern we can see, of Gestalt and of our innate propensity to interpret stimuli as faces based on minimal cues [23, 24]. As a matter of fact, we are hard-wired to perceive faces from several random patterns: see, e.g., Fig. 7 which surprisingly shows the perception of at least three different Shroud-like faces and of a bear-like nose in the same Fig. 5a. The examples in Fig. 7 illustrate how it is easy to deceive our perception, and how effective is our brain to produce false positives which allow to see faces and figures that do not exist.

\section{Conclusion}

This paper summarizes the overall research work on Shroud-related studies done in the last years at the ENEA Research Centre of Frascati. In addition to a summary of prior results, we have presented unpublished results that complete the outcomes previously scattered in a number of papers $[8,10,11,14,19]$.

Our laser irradiations of linen fabrics showed the ability of ultra-short, high intensity UV and VUV radiation to reproduce many peculiar aspects of the microscopic complexity of the TS images, as detailed in [11] and briefly summarized in Sect. 2.2 and Sect. 2.3. 
We have used the laser beam as a contactless brush to draw the face of the man of the TS, stylized according to the binary logo of the 2010 Shroud exposition. The result of this preliminary attempt is quite interesting, see Sect. 2.4.

The different coloration results obtained by diverse laser irradiation parameters (wavelength, intensity, fluence, repetition rate, number of shots) allowed to recognize the photochemical reactions that may have contributed to the Shroud-like coloration, thus offering hints to plan the long-term conservation of the cloth and of the image on it, see Sect. 3 and [14].

Our study of the misleading effect of digital image processing of photographs of low-contrast images confirmed that there is a narrow boundary between image enhancement and manipulation. In particular, data and analyses in Sect. 4 demonstrate the lack of spatial correlation between the face on the TS and the alleged "hidden" face underside which was "discovered" after a heavy image processing of a photograph printed in a book. This lack of correlation calls the existence of a face underside into serious doubt, and supports the results of a previous in-depth analysis [22] which showed the absence of any face on the backside of the TS. In addition, our results in [19] show that some alleged inscriptions hidden on the TS [15] are unlikely to exist.

This paper summarizes more than eight years of experiments, calculations and discussions. Here we wish to thank the Colleagues who gave a contribution to different phases of this long-term work, including, in alphabetical order, G. Baldacchini, G. Fanti, E. Marinelli, E. Nichelatti, A. Santoni, B. Schwortz. Without their skill, ideas and collaborative approach the successful results summarized in this paper could not have been achieved.

\section{References}

[1] The official summary of the final report of STuRP can be read at the webpage http://www. shroud.com/78conclu.htm

[2] E. Jumper, A.D. Adler, J.P. Jackson, S.F. Pellicori, J.H. Heller, J.R. Druzik, Archaeological Chemistry III: ACS Advances in Chemistry 205 (American Chemical Society, Washington, 1984), pp. $447-476$

[3] A complete list of scientific papers published by the STuRP team can be found in http: //www. shroud.com/78papers.htm

[4] J.P. Jackson, E.J. Jumper, and W.R. Ercoline, Appl. Opt. 23, 2244-2270 (1984)

[5] G. Fanti, J. Botella, P, Di Lazzaro, R. Schneider, N. Svensson, J. Im. Sci. Technol. 54, 040201-(8) (2010)

[6] The most interesting and best documented attempt to reproduce a life-size Shroud-like image is described in L. Garlaschelli, J. Im. Sci. Technol. 54, 040301-040301(14) (2010). References therein offer a comprehensive list of other attempts. See a critical review of this paper in: T. Heimburger, G. Fanti, Proceedings of the International Workshop on the Scientific approach to the Acheiropoietos Images, IWSAI 2010, (ENEA, 2010) pp. 19-28 www . acheiropoietos.info/proceedings/proceedings.php

[7] Web page of Shroud-related studies of the Excimer Laser Laboratory: http://www.frascati.enea.it/fis/lac/excimer/sindone/sindone.html The overall activities of our Lab can be found at the website www. frascati.enea.it/fis/lac/excimer/ labeccimeri_eng.html

[8] P. Di Lazzaro, D. Murra, E. Nichelatti, A Santoni, G. Baldacchini, J. Im. Sci. Technol. 54, 040302-(6) (2010)

[9] R. Rogers, A. Arnoldi, www . shroud.com/pdfs/rogers2.pdf (2002). In this document, Rogers and Arnoldi wrote: "Very intense, 50-ns-long bursts of UV ablated the cloth surface, and the 
samples were reduced to a cloud of very fine particles. We could not get a color with a flash of light." In another document (www.shroud.com/pdfs/rogers6.pdf (2004)) Rogers criticised the radiative hypothesis of Jackson published in Shroud Spectrum International 34, 3-29 (1990) and wrote: "Experiments we did with pulsed ultraviolet lasers on linen resulted in ablation and destructive shock waves. Samples often were converted into a little amorphous powder and gas, ablate and convert linen into little amorphous powder and gas". One of the authors (PDL) in 1991 was invited by Robert (Bob) Sze to visit his Excimer Laser Group at Los Alamos National Laboratory (LANL). All the "non restricted" experiments of Sze's group were equipped with excimer lasers emitting laser pulses each having a width in the 50-150-ns range, like those emitted by the Hercules ENEA excimer laser. Rogers was working at LANL, and we can speculate he asked Sze's group to make excimer irradiations of linen, and that they used the $50-150 \mathrm{~ns}$ laser pulsewidth they had at hands. As explained in Sect. 2.1, we obtained the same ablation results when irradiating linens with the $120 \mathrm{~ns}$ long Hercules laser pulses. The results described in Sects. 2.1 and 2.2 which are obtained using shorter-than-30-ns UV and VUV laser pulses demonstrate the paramount importance of the laser pulsewidth to achieve a Shroud-like coloration of linen

[10] G. Baldacchini, P. Di Lazzaro, D. Murra, G. Fanti, Appl. Opt. 47, 1278-1283 (2008)

[11] P. Di Lazzaro, D. Murra, E. Nichelatti, A Santoni, G. Baldacchini, Appl. Opt. 51, 8567-8578 (2012)

[12] M. Yatagai, S.H. Zeronian, Cellulose 1, 205-214 (1994)

[13] Phisik Instrumente model M-521. Each stage was independently driven by a Mercury C-863 DC motor controller

[14] P. Di Lazzaro, D. Murra, A. Santoni, E. Nichelatti, EAI special issue on Knowledge, Diagnostics and Preservation of Cultural Heritage, 89-94 (2012). http: //www . enea . it/it/produzionescientifica/pdf-eai/speciale-cultural-heritage/14-the-conservation-pdf

[15] A. Marion, Opt. Eng. 37, 2308-2313 (1998)

[16] A.D. Whanger, M. Whanger, Appl. Opt. 24, 766-772 (1985)

[17] A. Danin, A.D. Whanger, U. Baruch, M. Whanger, Flora of the Shroud of Turin (Missouri Botanical Garden Press, St. Louis MO, 1999)

[18] G. Fanti, R. Maggiolo, J. Opt. A: Pure and Applied Optics 6, 491-503 (2004)

[19] P. Di Lazzaro, D. Murra, B. Schwortz, Pattern Recognition 46, 1964-1970 (2013)

[20] G. Kanizsa, Scientific American 234, 48-52 (1976)

[21] See, e.g., J. Voss, K. Federmeier, K. Paller, Cerebral Cortex 22, 2354-2364 (2012). For an elementary introduction, see http://en.wikipedia.org/wiki/Apophenia

[22] N. Balossino, Sindon 19, 57-69 (2003)

[23] R. Palermo, G. Rhodes, Neuropsychologia 45, 75-92 (2007)

[24] A J. Calder, G. Rhodes, M. Johnson, The Oxford Handbook of Face perception (Oxford University Press, 2011) 